\title{
Radioterapia estereotáctica
}

\section{Stereotactic radiation therapy}

\author{
J.J. Aristu' ${ }^{1}$, R. Ciérvide, J. Guridi ${ }^{2}$, M. Moreno ${ }^{1}$, L. Arbea ${ }^{1}$, J.D. Azcona ${ }^{1}$, L.I. Ramos ${ }^{1}$, \\ J.L. Zubieta ${ }^{3}$
}

\section{RESUMEN}

La radioterapia con técnica estereotáctica es una modalidad de radioterapia externa que utiliza un sistema de coordenadas tridimensionales independientes del paciente para la localización precisa de la lesión. También se caracteriza porque los haces de irradiación son altamente conformados, precisos y convergentes sobre la lesión que hacen posible la administración de dosis muy altas de radioterapia sin incrementar la irradiación de los órganos o estructuras sanas adyacentes. Cuando el procedimiento se realiza en una sesión de tratamiento se denomina radiocirugía y si se administra en varias sesiones se denomina radioterapia estereotáctica. Se precisa de sistemas de fijación e inmovilización del paciente especiales (guías o marcos estereotácticos) y dispositivos de radioterapia capaces de generar haces muy conformados (acelerador lineal, gammaknife, cyberknife, tomoterapia, ciclotrones). La radioterapia estereotáctica moderna utiliza marcas radioopacas intratumorales o sistemas de imágenes de TAC incluidos en el dispositivo de irradiación, que permiten una precisa localización de las lesiones móviles en cada sesión de tratamiento. Además, los avances tecnológicos hacen posible coordinar los movimientos de la lesión en la respiración con la unidad de radioterapia (gaiting y tracking) de forma que pueden estrecharse al máximo los márgenes y por lo tanto excluir un mayor volumen de tejido sano

La radiocirugía está indicada principalmente en lesiones cerebrales benignas o malignas menores de 3-4 centímetros (malformaciones arteriovenosas, neurinomas, meningiomas, metástasis cerebrales) y la radioterapia estereotáctica se administra fundamentalmente en tumores de localización extracraneal que requieran una alta conformación y precisión como cáncer precoz de pulmón inoperable y metástasis hepáticas.

Palabras clave. Radiocirugía. Radioterapia estereotáctica. Tumores cerebrales. Cáncer de pulmón. Metástasis hepáticas.

\begin{abstract}
Stereotactic radiotherapy is an external radiation modality that uses a system of three dimensional references independent of the patient to achive a precise location of the lesion. Stereotactic radiotherapy generate highly conformal, precisely focused radiation beams to administer very high doses of radiation without increasing the radiation to healthy surrounding organs or structures. When the procedure is carried out in one treatment session the procedure is termed radiosurgery, and when the treatment is administered in several fractions, the radiation modality is termed stereotactic radiotherapy. Special systems of patient immobilization (guides or stereotactic frames) are required together with radiotherapy devices capable of generating conformal beams (lineal accelerator, gammaknife, cyberknife, tomotherapy, cyclotrons). Modern stereotactic radiotherapy techniques employ intratumoural radio-opaque fiducials or CT image systems included in the irradiation device, which make possible a precise location of mobile lesions in each treatment session. Besides, technological advances permit breathing synchronized radiation (gating and tracking) for maximum tightening of margins and excluding a greater volume of healthy tissue.

Radiosurgery is mainly indicated in benign or malign cerebral lesions less than 3-4 centimetres (arteriovenous malformations, neurinomas, meningiomas, cerebral metastases) and stereotactic radiotherapy is basically administered in tumours of extracraneal location that require high conformation and precision, such as inoperable early lung cancer and liver metastasis.
\end{abstract}

Key words. Radiosurgery. Stereotactic radiotherapy. Cerebral tumours. Lung cancer. Hepatic metastasis.
1. Servicio de Oncología Radioterápica. Departamento de Oncología.

Clínica Universidad de Navarra.

2. Servicio de Neurocirugía.

Departamento de Neurología,

Neurocirugía y Neurofisiología.

Clínica Universidad de Navarra.

3. Departamento de Radiología.

Clínica Universidad de Navarra.

\author{
Correspondencia \\ José Javier Aristu Mendioroz \\ Servicio de Oncología Radioterápica \\ Clínica Universidad de Navarra \\ Pío XII, 36 \\ 31008 Pamplona \\ Tfno. 948255400 \\ Fax 948255500 \\ E-mail: jjaristu@unav.es
}




\section{INTRODUCCIÓN}

La radioterapia estereotáctica es una técnica de radioterapia externa que a través de un sistema de coordenadas tridimensionales independientes del paciente permite una precisa localización de la lesión. La radioterapia externa se administra en forma de haces de irradiación altamente conformados, precisos y convergentes sobre la lesión que hacen posible la administración de dosis muy altas de radioterapia sin incrementar la irradiación de los órganos o estructuras sanas adyacentes ${ }^{1}$. Se utiliza principalmente para tratar lesiones cerebrales utilizando una dosis única de radioterapia o en localizaciones extracraneales como pulmón e hígado de manera fraccionada.

\section{SECUENCIA DEL PROCEDIMIENTO}

\section{Inserción de una guía o marco estereotáctico}

Se precisa la colocación de una guía (guía estereotáctica) con el objeto de inmovilizar al paciente y definir unos puntos de referencia externos. Esta guía puede ser fija (para un solo tratamiento) o reposicionable (para tratamiento fraccionado). En el caso de un único tratamiento, sobre el cerebro se utiliza una guía estereotáctica inserta en el cráneo del paciente a través de fijaciones atornilladas en la tabla externa de la calota del paciente. Además este marco estereotáctico se utiliza para colocar un sistema de puntos de referencia que permiten una localización exacta de la lesión en los tres planos del espacio. En la radioterapia estereotáctica extracraneal suelen utilizarse inmovilizadores corporales (colchones de vacío o de resinas) dentro de un marco o guía corporal rígida donde se sitúan los puntos de referencia, aunque como se verá más adelante este tipo de inmovilización está evolucionando hacia la práctica de marcadores internos o adquisición de imágenes tomográficas en la misma sala de tratamiento para verificación de la posición del tumor previa al tratamiento.

\section{Adquisición de imágenes}

Posteriormente se adquieren imágenes de TAC en condiciones estereotácticas (incluyendo los puntos de referencia) y habitualmente imágenes de RM para su fusión con las imágenes de TAC y reconstrucción tridimensional del paciente. También pueden utilizarse imágenes de PET cuando la delimitación de la lesión es dificultosa con TAC-RM.

\section{Planificación}

En el planificador se contornean la lesión y los órganos y estructuras sanas adyacentes radiosensibles. Esto permite reconstruir al paciente en las tres dimensiones del espacio y diseñar de forma virtual el plan de tratamiento más adecuado (Fig. 1). En los planificadores más modernos el sistema de planificación genera de forma semi-automática varios planes teniendo en cuenta distintas preferencias. Se identifica y selecciona el plan de irradiación que mejor se adapta a la morfología de la lesión y administra menos dosis de radioterapia sobre los órganos y estructuras sanas adyacentes. Con posterioridad se realiza la prescripción de la dosis de irradiación que debe abarcar toda la lesión y se optimiza el plan.

\section{Verificación}

Antes de iniciar el tratamiento debe comprobarse la correcta disposición de ciertos componentes mecánicos del acelerador lineal y del haz de irradiación. El proceso de control de calidad consiste en la verificación de la correcta alineación de los láseres que sirven para el posicionamiento preciso del paciente antes del tratamiento y de la adecuada relación del haz de irradiación con respecto a un punto cuando gira el cabezal del acelerador. También, si se utiliza multiláminas o micromultiláminas para la conformación del haz debe verificarse su correcto recorrido en el movimiento de las láminas. 

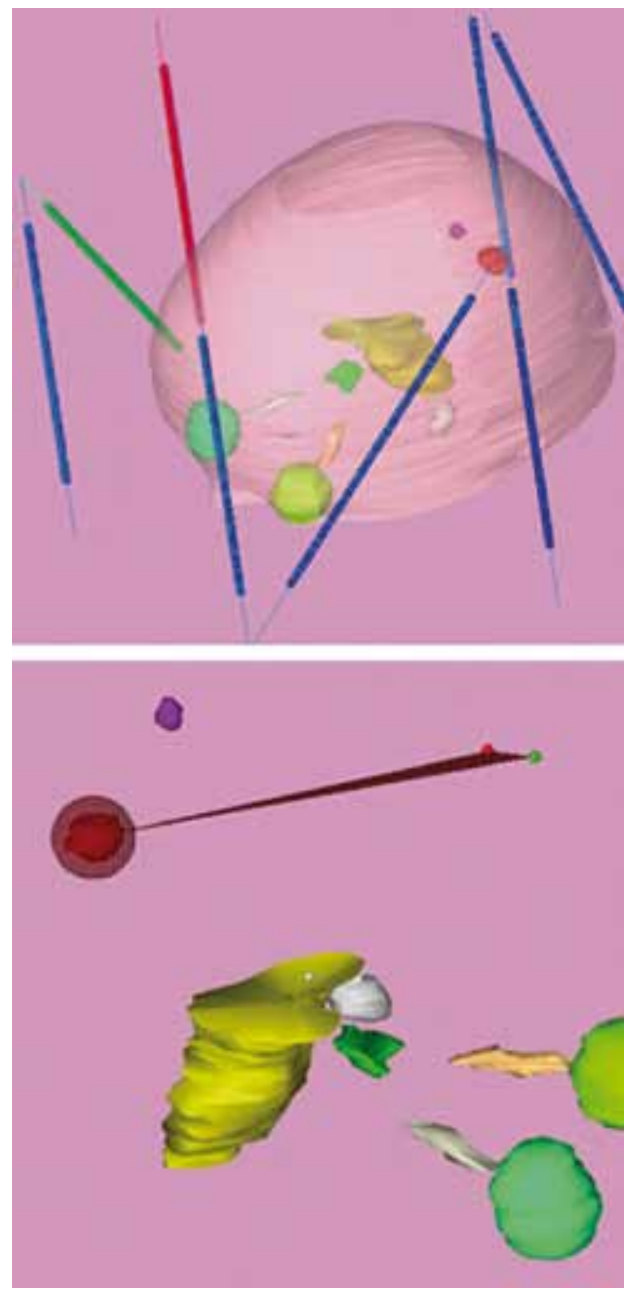

Figura 1. Representación tridimensional de los órganos a riesgo (ojos, nervios ópticos, quiasma y tronco cerebral) y 2 metástasis cerebrales contorneados en el planificador de radiocirugía.

\section{Administración del tratamiento}

El paciente es posicionado en la mesa de tratamiento de forma idéntica a la posición establecida en la TAC para la adquisición de imágenes. Las coordenadas numéricas estereotácticas del centro de la lesión, calculadas por el planificador y referenciadas en los tres planos del espacio, son localizadas en una escala calibrada en décimas de milímetro sobre el marco estereotáctico. Estas referencias se hacen coin- cidir con los láseres que apuntan al isocentro mecánico del acelerador lineal (punto de rotación del cabezal del acelerador). El tratamiento puede realizarse utilizando distintas técnicas dependiendo del dispositivo de radioterapia (ver más adelante).

El tiempo invertido desde la inserción o diseño del marco estereotáctico hasta la finalización del tratamiento suele oscilar entre 3 y 6 horas dependiendo de la complejidad del proceso, correspondiendo 30 a 90 minutos el tiempo real de utilización del dispositivo de radioterapia. El paciente puede ser tratado de forma ambulatoria.

\section{TIPOS DE RADIOTERAPIA ESTEREOTÁCTICA}

\section{Según el fraccionamiento}

Cuando se administra una única fracción el procedimiento se denomina radiocirugía o radiocirugía estereotáctica y si se utiliza más de una fracción la técnica se denomina radioterapia estereotáctica.

\section{Radiocirugía}

La radiocirugía o radiocirugía estereotáctica es una técnica especial de radioterapia externa que administra una de dosis única de irradiación utilizando un sistema de coordenadas estereotáctico, con alto gradiente (en pocos milímetros de tejido la dosis de irradiación decae bruscamente) y precisión sobre un volumen pequeño (menor de 3-4 centímetros de diámetro) y generalmente localizado en el cerebro. Desde el año 1951 en el que Lars Leksell desarrolló y definió la técnica ${ }^{2}$ hasta la actualidad, la radiocirugía ha evolucionado notablemente tanto en los sistemas de planificación como en los dispositivos empleados para la administración de la irradiación. Se trata de una técnica multidisciplinar en la que se requiere la colaboración de médicos neurocirujanos, neurorradiólogos, físicos médicos, oncólogos radioterápicos, enfermeras y técnicos en radioterapia.

En la radiocirugía se precisa de una firme inmovilización del paciente y para ello 
se utilizan guías estereotácticas rígidas que se fijan al paciente mediante la inserción de cuatro puntos de anclaje en la calota que se retiran al finalizar el procedimiento (Fig. 2a). El margen de error aceptado es menor a $1 \mathrm{~mm}$. En muy raras ocasiones la radiocirugía se utiliza para el tratamiento de lesiones extracerebrales.

\section{Radioterapia estereotáctica}

La radioterapia estereotáctica comparte con la radiocirugía la alta precisión y el gradiente de la dosis pero la administración de la irradiación se realiza de forma fraccionada. Explota las ventajas radiobiológicas del fraccionamiento de la dosis, mejorando la tolerancia del tejido sano adyacente. La radioterapia estereotáctica se indica muy frecuentemente en el tratamiento de lesiones extracerebrales, fundamentalmente pulmón e hígado (radioterapia estereotáctica corporal). Se han utilizado guías corporales con referencias estereotácticas visibles en una carcasa rígida en la que se posiciona el paciente utilizando un molde corporal com- binado o no con sistemas de vacío. Con la introducción de la radioterapia guiada por la imagen, la práctica actual sin embargo ha cambiado y se fundamenta en la inserción de marcas radioopacas intratumorales o próximas a él para la localización exacta del target antes del cada tratamiento. Se precisan sistemas de CT o de kilovoltaje en la misma sala de tratamiento y sincronizar con la irradiación o minimizar los movimientos internos del volumen target. En el cerebro, la radioterapia estereotáctica se utiliza para tratar lesiones menores de 3-4 centímetros muy próximas a estructuras radiosensibles o lesiones mayores de 4 centímetros. Para la inmovilización de la cabeza del paciente e inserción de los puntos de referencia estereotácticos se utiliza una guía estereotáctica reposicionable que se posiciona en la cabeza del paciente diariamente en cada sesión de tratamiento. Son guías no cruentas que se adaptan y fijan al paciente mediante distintos dispositivos de sujeción como moldes dentales o material termoplástico (Fig. 2b). El margen de error aceptado es menor de $2 \mathrm{~mm}$.

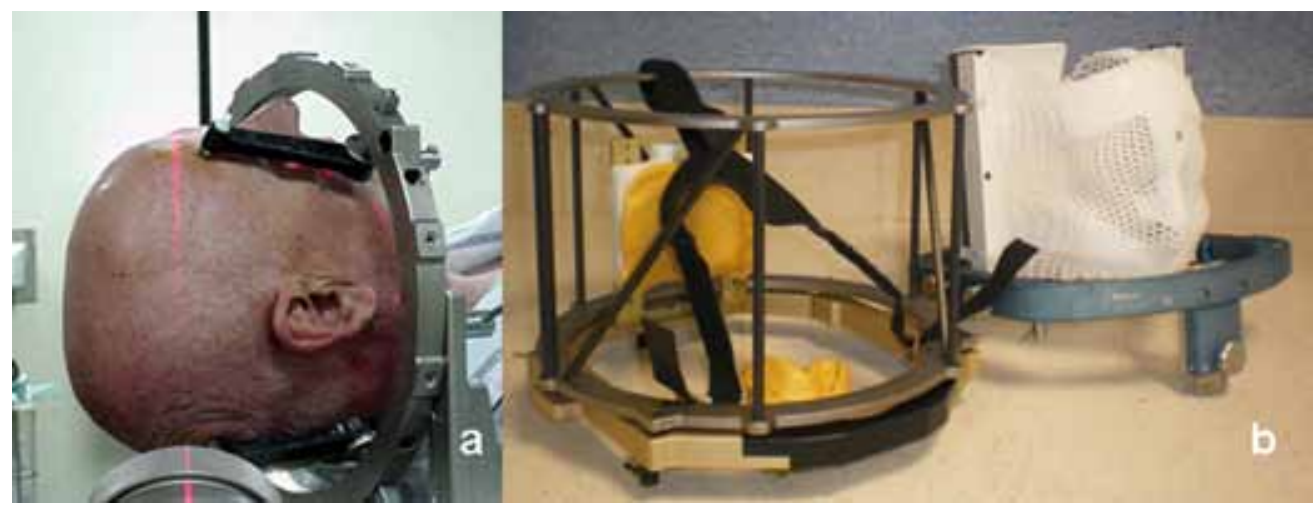

Figura 2. Guía estereotáctica BRW para radiocirugía enclavada en el cráneo de un paciente (a) y guías estereotácticas para radioterapia estereotáctica (reposicionables) (b).

\section{Según la localización de la lesión}

\section{Radiocirugía y radioterapia estereotáctica craneal}

El tratamiento con técnicas estereotácticas en el cerebro se basa fundamentalmen- te en radiocirugía por distintas razones. La primera es la facilidad para inmovilizar y reproducir con alta precisión la misma posición del cráneo y el poco movimiento o desplazamiento interno del cerebro. Estas características hacen muy factible confinar 
al máximo los campos de irradiación con mucha seguridad y excluir un mayor volumen de tejido sano. La segunda ventaja es la posibilidad de administrar una dosis única y alta de irradiación que radiobiológicamente es muy eficaz en tejidos de proliferación lenta, lesiones muy frecuentemente tratadas con radiocirugía (malformaciones arteriovenosas, neurinomas, meningiomas, etc). El tratamiento con radioterapia estereotáctica en el cerebro se reserva para lesiones mayores de 3 centímetros, lesiones de cualquier tamaño situadas a menos de 2 mm de estructuras muy sensibles a dosis altas de radioterapia como nervios ópticos, quiasma o tronco cerebral y en técnicas de sobreimpresión principalmente de tumores en niños para excluir el mayor volumen de tejido cerebral sano fuera de las dosis altas de irradiación.

\section{Radiocirugía y radioterapia estereotáctica extracraneal}

La técnica estereotáctica de elección en localizaciones extracraneales es la radioterapia estereotáctica. Es muy comprometido administrar con seguridad una dosis única de irradiación en una lesión hepática o pulmonar en movimiento. Por otra parte es también más dificultoso reproducir con exactitud la posición del tórax, abdomen o pelvis de un paciente por carecer de la rigidez característica del cráneo. Por último, las lesiones extracraneales subsidiarias de tratamiento estereotáctico son generalmente de mayor volumen que las tratadas en el cerebro y por lo tanto la probabilidad de complicaciones severas con dosis muy altas de irradiación son más elevadas.

Aunque en la radioterapia estereotáctica se utilizan marcos corporales rígidos e indexados para la localización del volumen diana que debe ser irradiado, la realidad es que con los avances tecnológicos disponibles actualmente para la visualización diaria de las lesiones tratadas antes y durante el tratamiento radioterápico, los marcos corporales están en desuso y por lo tanto no se utilizan referencias externas. De esta forma el término "radioterapia estereotáctica" se usa de forma incorrecta y suele asociarse a dosis altas de radioterapia guiadas por la imagen administradas generalmente en 3-5 fracciones sin necesidad de coordenadas estereotácticas.

\section{Según el dispositivo de irradiación}

Las unidades de radioterapia más extendidas para el tratamiento con técnicas estereotácticas son la gammaknife y los aceleradores lineales. La gammaknife, es una unidad de irradiación compuesta por múltiples fuentes de ${ }^{60} \mathrm{Cobalto}$ (genera rayos gamma que son fotones de 1,25 Mev) en forma de haces circulares convergentes en un punto y está dedicada exclusivamente a tratamientos con radiocirugía de lesiones cerebrales. La gammaknife no puede utilizarse para radioterapia estereotáctica. Durante el tratamiento con gammaknife, el equipo permanece estático y la distribución irregular de la dosis se obtiene por la suma de varios haces de irradiación adyacentes.

Los aceleradores lineales generan rayos X (fotones de 4-21 Mev) y electrones a partir de diferencias de potencial eléctrico (no utilizan material radiactivo) y son dispositivos de radioterapia externa que se utilizan de forma convencional para el tratamiento del cáncer y además pueden ser adaptados para radiocirugía o radioterapia estereotáctica. A diferencia de la gammaknife, los aceleradores lineales se mueven alrededor del paciente durante el tratamiento, pueden realizar procedimientos de radiocirugía y de radioterapia estereotáctica y pueden tratar lesiones extracerebrales. El tratamiento se administra mediante múltiples campos estáti$\cos \mathrm{o}$ arcos rotacionales del cabezal del acelerador que concentran la dosis de irradiación en la lesión mientras se minimiza al máximo la cantidad de irradiación en el tejido cerebral sano adyacente (Fig. 3). La distribución de la dosis se conforma utilizando colimadores circulares, micromultiláminas con campos fijos o dinámicos e IMRT. 

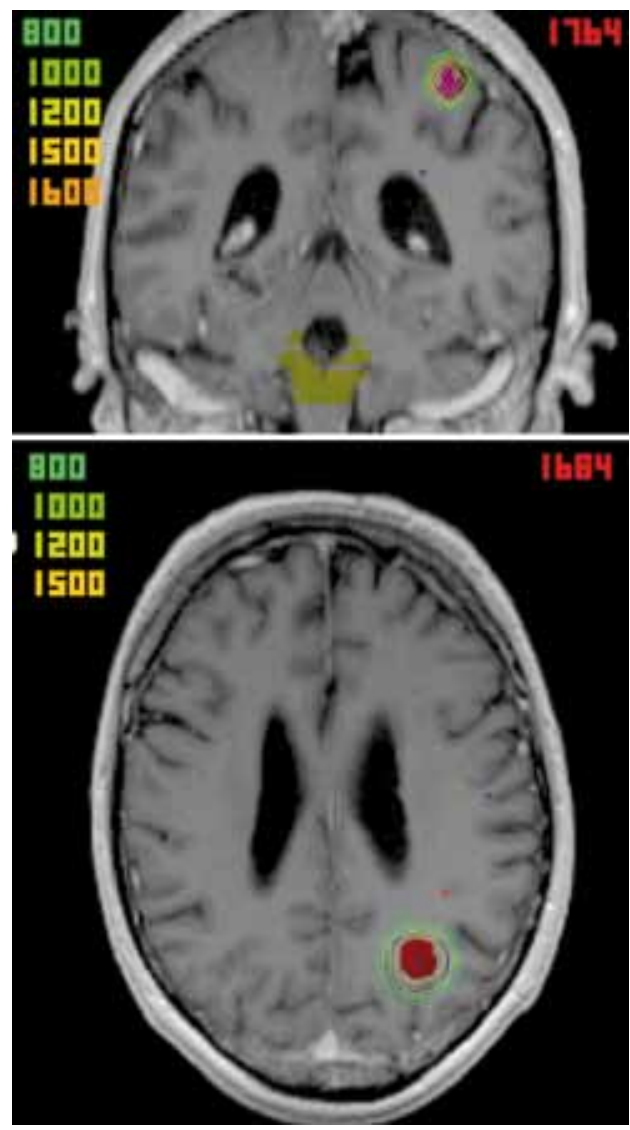

Figura 3. Distribución dosimétrica de un tratamiento con radiocirugía. Puede observarse la alta precisión y gradiente de la dosis de irradiación concentrando la dosis en la lesión y respetando el tejido cerebral sano.

Actualmente existe en el mercado un dispositivo denominado Cyberknife (acelerador lineal con un brazo robotizado) que permite realizar el tratamiento con más grados de libertad en cualquier parte del cuerpo. La tomoterapia, acelerador lineal integrado en un TAC, también puede adaptarse para realizar tratamientos con técnicas estereotácticas.

El tratamiento con radiocirugía en ciclotrones (generadores de protones) está muy restringido a unos pocos centros en el mundo y se utiliza fundamentalmente para el tratamiento de tumores pediátricos, de base de cráneo y paraespinales.

\section{INDICACIONES Y RESULTADOS DE RADIOTERAPIA CON TÉCNICAS ESTEREOTÁCTICAS}

Nos referiremos a las indicaciones de radiocirugía craneal y radioterapia estereotáctica extracraneal o corporal porque incluyen la mayoría de los pacientes tratados con técnicas estereotácticas.

\section{Radiocirugía craneal}

\section{Malformaciones arteriovenosas (MAV)}

Las MAV pueden tratarse mediante observación, cirugía, embolización endovascular (para reducir el volumen o flujo de la $\mathrm{MAV}$ ) y radiocirugía de forma exclusiva o en combinación. El riesgo de hemorragia sin tratamiento se calcula en un $2,4 \%$ anual. Las MAV candidatas a radiocirugía deben tener menos de $3 \mathrm{~cm}$ de diámetro $(0,1-10 \mathrm{cc})$ aunque las MAV entre 10-15 cc pueden tratarse con radiocirugía subdividiendo la MAV volumétricamente y tratando cada subvolumen de manera independiente siempre y cuando no exista una estrategia terapéutica mejor. Para la planificación de la radiocirugía se requiere integrar en el planificador las imágenes de TAC y de angiografía en condiciones estereotácticas. La dosis administrada al margen de la MAV oscila entre 16-25 Gy dependiendo del volumen y localización (las MAV profundas requieren dosis bajas por el riesgo de complicaciones). Generalmente se administra tras el tratamiento una dosis de dexametasona o metilprednisolona y los pacientes pueden seguir recibiendo su medicación habitual. La obliteración gradual de la MAV se observa en imágenes de RM realizadas a intervalos de 6 meses durante los 3 primeros años. Si transcurrido este periodo se observa una obliteración completa de la MAV en la RM debe realizarse una angiografía para confirmar la obliteración. Si después de tres años la angiografía demuestra un cierre incompleto de la MAV se recomienda repetir la radiocirugía. En el tiempo de latencia entre la radiocirugía y el cierre de la MAV el riesgo de hemorragia no se elimina sino que se reduce aproximadamente un $50 \%$. Entorno a un $75 \%$ de las MAV se 
cierran completamente después de radiocirugía ${ }^{3}$, las MAV pequeñas un $95 \%$ y las voluminosas $50 \%$. La probabilidad de observar cambios en las secuencias potenciadas en $\mathrm{T} 2$ en la RM se relacionan con el volumen de tejido cerebral sano rodeando la MAV que recibe 12 Gy o más ${ }^{4}$. Los efectos adversos precoces son raros: cefalea por la inserción de la guía, crisis comiciales en MAV corticales especialmente si los pacientes presentan antecedentes de crisis. Los efectos adversos a largo plazo incluyen hemorragia (incluso después del cierre angiográfico de la MAV), edema, necrosis, tumores inducidos $(0,001 \%)$ y formación quística $(4,7 \%)$.

\section{Neurinoma del acústico}

Actualmente la radiocirugía es una alternativa al tratamiento con microcirugía del neurinoma del VIII par craneal. Los candidatos ideales son pacientes con neurinoma de tamaño pequeño o medio (menos de $3 \mathrm{~cm}$ de diámetro) y sin comprensión sintomática del tronco cerebral. También está indicada en pacientes con neurinoma residual o recurrente después de cirugía.

La radiocirugía es una técnica mínimamente invasiva especialmente apropiada cuando se persigue una alta probabilidad de preservación de funciones neurológicas (coclear y facial) y una alta tasa de control tumoral. El control local con esta técnica alcanza el 95-98\%, preserva la audición en el $60-70 \%$ de los casos y preserva el nervio facial y trigémino en más del $95 \%$ de los pacientes. Se ha demostrado respecto a la microcirugía una menor tasa de neuropatía facial y trigeminal, mejor preservación de la audición medible y resultados equivalentes en el mantenimiento de una audición útil y control tumoral. Adicionalmente puede añadirse un beneficio por un menor coste y menor estancia hospitalaria, reducción del riesgo de sangrado, problemas anestésicos, infecciones y otros efectos secundarios transitorios o permanentes a una cirugía abierta ${ }^{5-7}$.

La dosis única óptima de irradiación ha ido disminuyendo en los últimos 10 años hasta situarse actualmente en 12-13 Gy con la que se obtiene un óptimo control local con mínimos efectos adversos. Se contemplan dosis más bajas en pacientes con NF2 o sordera contralateral por otras causas en los que la preservación de la audición es más crítica.

\section{Meningioma}

Aunque la cirugía continúa siendo el tratamiento convencional en el meningioma, la radiocirugía es la modalidad terapéutica de elección en el tratamiento de los meningiomas intracraneales benignos, bien delimitados, menores de 3 centímetros y/o inaccesibles a cirugía, recurrentes tras cirugía o de histología atípica ${ }^{8-10}$.

El control local obtenido con radiocirugía es equivalente al observado con resecciones Grado 1 de Simspson y superior cuando se compara con resecciones iguales o mayores a Grado 2 de Simpson ${ }^{11}$.

La radiocirugía ha demostrado ser un tratamiento seguro y efectivo, especialmente en localizaciones de difícil acceso quirúrgico como el seno cavernoso o región petroclival ya sea como tratamiento primario o después de resección incomple$\mathrm{ta}^{9,12}$. Las secuelas neurológicas descritas postradiocirugía de meningiomas localizados en la base del cráneo consisten en edema con cefalea, empeoramiento transitorio de síntomas previos que habitualmente responden a corticoides, alteraciones del nervio trigémino y déficits campimétricos temporales o permanentes ${ }^{12}$. La tasa actual de cualquier complicación a 5 y 10 años es el $9 \%$. Es frecuente observar una mayor tasa de edema en meningiomas de la convexidad, parasagitales y de la hoz cerebral tratados con radiocirugía ${ }^{13,14}$. El control local alcanzado es el $90 \%$ en la mayoría de los estudios y las complicaciones registradas menos del $5 \%$ si la dosis prescrita al margen es menor de $15 \mathrm{~Gy}^{15}$.

\section{Metástasis cerebrales}

La radiocirugía en metástasis cerebrales está indicada en pacientes con buen estado general menores de 65 años, tumor 
primario controlado y ausencia o control de la enfermedad oligometastásica extracerebral $^{16,17}$. Aunque habitualmente se tratan con radiocirugía entre 1-4 metástasis cerebrales, se desconoce el número adecuado de metástasis que se beneficia del tratamiento radioquirúrgico. La radiocirugía se utiliza también en el tratamiento de metástasis recurrentes después de cirugía o radioterapia holocraneal. El rango de dosis establecido de 14-24 Gy se basa en la localización del tumor (próxima a áreas elocuentes cerebrales), volumen tumoral (metástasis hasta $4 \mathrm{~cm}$ de diámetro), tratamiento radioterápico previo y estimación del riesgo de efectos adversos. La tasa de control local a 12-18 meses con radiocirugía exclusiva es $75 \%$ y cuando se asocia con radioterapia holocraneal alcanza el $80-85 \%{ }^{18}$. Se observan muy pocos efectos adversos agudos, describiéndose fatiga, pérdida de pelo parcheada y transitoria y convulsiones en pacientes con historia previa de convulsiones o metástasis corticales. El efecto tardío más severo y común es la radionecrosis aunque el riesgo estimado de radionecrosis sintomática es menor del $5 \%{ }^{8,19,20}$. La radionecrosis genera edema y efecto masa que puede producir déficits neurológicos y convulsiones. El tratamiento inicial es la administración de corticoides y en caso de no respuesta puede recomendarse resección quirúrgica para eliminar el efecto masa.

La radiocirugía puede utilizarse como tratamiento exclusivo o en combinación con cirugía o radioterapia holocraneal. El tratamiento con radiocirugía más radioterapia holocraneal frente a radioterapia holocraneal exclusiva mejora significativamente el control local en pacientes con 4 metástasis cerebrales o menos, acorta el periodo de administración de corticoides y mejora el índice Karnofsky a los 6 meses. Además el tratamiento combinado en pacientes seleccionados con metástasis cerebral única incrementa significativamente la supervivencia ${ }^{8,19}$.

La omisión de radioterapia holocraneal en pacientes con 1-3 metástasis cerebrales no disminuye la supervivencia aunque in- crementa la recurrencia local y cerebral de la enfermedad ${ }^{18}$.

\section{Radioterapia estereotáctica corporal}

\section{Cáncer de pulmón}

Muchos pacientes con tumores no microcíticos pulmonares precoces, periféricos, no son candidatos a resección quirúrgica por pobre función pulmonar u otras comorbilidades. En la última década un número creciente de instituciones han adquirido experiencia en el tratamiento de estas lesiones periféricas con radioterapia estereotáctica. Aunque en un principio no se incluyeron tumores centrales por el riesgo de toxicidad sobre estructuras mediastínicas, actualmente se han activado estudios fase I de escalada de dosis a partir de los resultados de factibilidad comunicados en series con un número limitado de pacientes.

Se ha empleado en los distintos estudios un fraccionamiento muy variable, demostrando una relación dosis-respuesta con un excelente control local utilizando dosis radiobiológicas muy altas ${ }^{21}$. Es conocido el fraccionamiento 20 Gy a 22 Gy x 3 de la Universidad de Texas ${ }^{22}$ o 12 Gy x 4 o 10 Gy x 5 de la experiencia japonesa ${ }^{23}$. A pesar de estas diferencias en el fraccionamiento, en la prescripción de la dosis y en las correcciones de la heterogeneidad del tejido, los diferentes estudios han demostrado un control local de alrededor de 90-95\%. La toxicidad mayor de grado 2 es infrecuente (2-5\%) siendo la neumonitis y la fractura costal los efectos adversos más observados. Se necesita un mayor seguimiento para confirmar estos datos y sobretodo conocer los resultados de distintos estudios randomizados aunque se sugiere que esta técnica es segura y altamente eficaz.

\section{Metástasis hepáticas}

La supervivencia a 5 años de pacientes seleccionados con enfermedad metastásica exclusiva hepática de cáncer de colon que llega a ser resecada es el $30-70 \%{ }^{24-26}$. Si la enfermedad hepática es irresecable, 
el remanente hepático es insuficiente o las condiciones médicas contraindican cirugía, se han explorado otras opciones terapéuticas como radiofrecuencia, embolización, quimioembolización o radioembilización. Por otra lado, existe una experiencia dilatada en el tratamiento con radioterapia externa de la totalidad del hígado con o sin sobreimpresión de las metástasis y se conoce la relación dosisrespuesta con la enfermedad hepática radioinducida (EHRI) ${ }^{27-30}$.

La no definición de una alternativa no quirúrgica de las metástasis hepáticas del cáncer de colon y los avances en escalada de dosis respecto a la tolerancia del hígado han conformado un escenario idóneo para la radioterapia estereotáctica como opción terapéutica de las metástasis hepáticas del cáncer de colon. Los resultados conocidos provienen de series uniinstitucionales con pocos pacientes y que utilizan diversos esquemas de hipofraccionamiento, 7,7-24 Gy en una fracción o 30 Gy en 4 fracciones ${ }^{31,32}, 60$ Gy en 3 fracciones ${ }^{33}$, 50 Gy en 5 fracciones $^{34}$ y dosis individualizada (mediana $36,6 \mathrm{~Gy}$ ) en 6 fracciones según el riesgo de $\mathrm{EHRI}^{35}$. El control local a los 18 meses en estas series se sitúa entorno al $70-90 \%$ y la mediana de supervivencia en 12-14 meses. El RTOG ha iniciado un estudio fase I (RTOG-0438) con el objetivo de identificar la máxima dosis tolerada hasta 50 Gy en 5 fracciones.

\section{CONSIDERACIONES FINALES}

La radioterapia externa basada en técnicas estereotácticas está adquiriendo un protagonismo relevante en el tratamiento de tumores benignos y malignos de volumen pequeño por su capacidad de generar una alta conformación de las dosis altas de irradiación minimizando la irradiación sobre órganos y estructuras sanas. El control local obtenido es muy alto y la toxicidad observada es baja. Actualmente el desarrollo tecnológico se orienta hacia el tratamiento de tumores primarios o enfermedad tumoral oligometastásica localizados en tórax, abdomen o pelvis utilizando pocas fracciones con el objetivo de inducir altas tasas de control local sin incrementar la toxicidad aguda o crónica y acortando de forma importante tiempo global de tratamiento.

\section{BIBLIOGRAFÍA}

1. LEKSELL L. Stereotactic radiosurgery. J Neurol Neurosurg Psychiatry 1983; 46: 797-803.

2. LEKSELL L. The stereotaxic method and radiosurgery of the brain. Acta Chir Scand 1951; 102: 316-319.

3. Pollock BE, Kondziolka D, Flickonger JC. Magnetic resonance imaging: an accurate method to evaluate arteriovenous malformations after stereotactic radiosurgery. J Neurosurg 1996; 85: 1044-1049.

4. Friedman WA, Bova FJ, Bollampally S, Bradshaw P. Analysis of factors predictive of success or complications in arteriovenous malformation radiosurgery. Neurosurgery 2003; 52 : 296-307.

5. Karpinos M, Teh BS, Zeck O, Carpenter LS, Phan C, MAI WY et al. Treatment of acoustic neuroma: stereotactic radiosurgery vs. microsurgery. Int J Radiat Oncol Biol Phys 2002; 54: 1410-1421.

6. Myrseth E, Moller P, Pedersen PH, Vassbotn FS, WentZEL-LARSEN T, LUND-JohANSEN M. Vestibular schwannomas: clinical results and quality of life after microsurgery or gamma knife radiosurgery. Neurosurgery 2005; 56: 927-935.

7. Pollock BE, Lunsford LD, Kondziolka D, FlickinGER JC, BissonetTe DJ, Kelsey SF et al. Outcome analysis of acoustic neuroma management: a comparison of microsurgery and stereotactic radiosurgery. Neurosurgery 1995; 36: 215224.

8. Kondziolka D, Patel A, Lunsford LD, Kassam A, FLICKINGER JC. Stereotactic radiosurgery plus whole brain radiotherapy versus radiotherapy alone for patients with multiple brain metastases. Int J Radiat Oncol Biol Phys 1999; 45: 427-434.

9. Lee JY, Niranjan A, McInerney J, Kondziolka D, FLICKINGER JC, LuNSFORD LD. Stereotactic radiosurgery providing long-term tumor control of cavernous sinus meningiomas. J Neurosurg 2002; 97: 65-72.

10. Subach BR, Lunsford LD, Kondziolka D, Maitz AH, FLICKINGER JC. Management of petroclival meningiomas by stereotactic radiosurgery. Neurosurgery 1998; 42: 437-443. 
11. Pollock BE, Stafford SL, Utter A, Giannini C, SCHREINER SA. Stereotactic radiosurgery provides equivalent tumor control to Simpson Grade 1 resection for patients with small- to medium-size meningiomas. Int J Radiat Oncol Biol Phys 2003; 55: 1000-1005.

12. Flickinger JC, Kondziolka D, Maitz AH, Lunsford LD. Gamma knife radiosurgery of imagingdiagnosed intracranial meningioma. Int J Radiat Oncol Biol Phys 2003; 56: 801-806.

13. Kim DG, Kim C, Chung HT, Paek SH, Jeong SS, HAN DH et al. Gamma knife surgery of superficially located meningioma. J Neurosurg 2005; 102 Suppl: 255-258.

14. Mindermann T, DE RO. The significance of tumor location for Gamma Knife treatment of meningiomas. Stereotact Funct Neurosurg 2004; 82: 194-195.

15. Marosi C, Hassler M, Roessler K, Reni M, Sant M, Mazza E et al. Meningioma. Crit Rev Oncol Hematol 2008; 67: 153-171.

16. Gaspar L, Scott C, Rotman M, Asbell S, Phillips T, WASSERMAN T et al. Recursive partitioning analysis (RPA) of prognostic factors in three Radiation Therapy Oncology Group (RTOG) brain metastases trials. Int J Radiat Oncol Biol Phys 1997; 37: 745-751.

17. Gaspar LE, Scott C, Murray K, Curran W. Validation of the RTOG recursive partitioning analysis (RPA) classification for brain metastases. Int J Radiat Oncol Biol Phys 2000; 47: 1001-1006.

18. Aoyama H, Shirato H, Tago M, Nakagawa K, ToyoDA T, Hatano K et al. Stereotactic radiosurgery plus whole-brain radiation therapy vs stereotactic radiosurgery alone for treatment of brain metastases: a randomized controlled trial. JAMA 2006; 295: 2483-2491.

19. Andrews DW, Scott CB, Sperduto PW, Flanders AE, Gaspar LE, Schell MC et al. Whole brain radiation therapy with or without stereotactic radiosurgery boost for patients with one to three brain metastases: phase III results of the RTOG 9508 randomised trial. Lancet 2004; 363: 1665-1672.

20. Auchter RM, Lamond JP, Alexander E, Buatti JM, Chappell R, Friedman WA et al. A multiinstitutional outcome and prognostic factor analysis of radiosurgery for resectable single brain metastasis. Int $\mathrm{J}$ Radiat Oncol Biol Phys 1996; 35: 27-35.

21. Dilling TJ, Hoffe SE. Stereotactic body radiation therapy: transcending the conventional to improve outcomes. Cancer Control 2008; 15: 104-111.
22. Timmerman R, McGarky R, Yiannoutsos C, Papiez L, Tudor K, DeLuca J et al. Excessive toxicity when treating central tumors in a phase II study of stereotactic body radiation therapy for medically inoperable early-stage lung cancer. J Clin Oncol 2006; 24: 4833-4839.

23. Onishi H, Shirato H, Nagata Y, Hiraoka M, FujlNo M, Gom K et al. Hypofractionated stereotactic radiotherapy (HypoFXSRT) for stage I non-small cell lung cancer: updated results of 257 patients in a Japanese multi-institutional study. J Thorac Oncol 2007; 2 (7 Suppl 3): S94-100.

24. Aloia TA, Vauthey JN, Loyer EM, Ribero D, PAWLIK TM, WEI SH et al. Solitary colorectal liver metastasis: resection determines outcome. Arch Surg 2006; 141: 460-466.

25. Сhoti MA, Sitzmann JV, Tiburi MF, SumetchotimeTha W, Rangsin R, Schulick RD et al. Trends in long-term survival following liver resection for hepatic colorectal metastases. Ann Surg 2002; 235: 759-766.

26. Fong Y, Cohen AM, Fortner JG, EnKer WE, TurNBULL AD, CorT DG et al. Liver resection for colorectal metastases. J Clin Oncol 1997; 15: 938-946.

27. Dawson LA, Normolle D, Balter JM, McGinn CJ, Lawrence TS, Ten Haken RK. Analysis of radiation-induced liver disease using the Lyman NTCP model. Int J Radiat Oncol Biol Phys 2002; 53: 810-821.

28. Jackson A, Ten Haken RK, Robertson JM, Kessler ML, KuTCHER GJ, LAWRENCE TS. Analysis of clinical complication data for radiation hepatitis using a parallel architecture model. Int J Radiat Oncol Biol Phys 1995; 31: 883-891.

29. Mohiuddin M, Chen E, Ahmad N. Combined liver radiation and chemotherapy for palliation of hepatic metastases from colorectal cancer. J Clin Oncol 1996; 14: 722-728.

30. Robertson JM, Sgueglia JB, Badger CA, Juston AC, Ballantyne J. Forensic applications of a rapid, sensitive, and precise multiplex off lysis of the four short tandem repeat loci HUMVWF31/A, HUMTH01, HUMF13A1, and HUMFES/FPS. Electrophoresis 1995; 16: 15681576.

31. Blomgren H, Lax I, Naslund I, Svanstrom R. Stereotactic high dose fraction radiation therapy of extracranial tumors using an accelerator. Clinical experience of the first thirty-one patients. Acta Oncol 1995; 34: 861-870.

32. Herfarth KK, Debus J, Lohr F, Bahner ML, Rhein $\mathrm{B}$, FRITZ $\mathrm{P}$ et al. Stereotactic single-dose ra- 
diation therapy of liver tumors: results of a phase I/II trial. J Clin Oncol 2001; 19: 164170.

33. Schefter TE, Kavanagh BD, Timmerman RD, CarDENES HR, BARON A, GASPAR LE. A phase I trial of stereotactic body radiation therapy (SBRT) for liver metastases. Int J Radiat Oncol Biol Phys 2005; 62: 1371-1378.
34. Katz AW, Carey-Sampson M, Muhs AG, Milano MT, Schell MC, OKuniefF P. Hypofractionated stereotactic body radiation therapy (SBRT) for limited hepatic metastases. Int J Radiat Oncol Biol Phys 2007; 67: 793-798.

35. DAwson LA, Eccles C, CRAIG T. Individualized image guided iso-NTCP based liver cancer SBRT. Acta Oncol 2006; 45: 856-864. 
